Comparison of liquid chromatography-mass spectrometry and direct infusion microchip electrospray ionization mass spectrometry in global metabolomics of cell samples

\author{
Pöhö, Päivi
}

2019-10-01

Pöhö , P , Lipponen , K , Bespalov , M M , Sikanen , T , Kotiaho , T \& Kostiainen , R 2019 , ' Comparison of liquid chromatography-mass spectrometry and direct infusion microchip electrospray ionization mass spectrometry in global metabolomics of cell samples ' , European Journal of Pharmaceutical Sciences , vol. 138 , 104991 . https://doi.org/10.1016/j.ejps.2019.104991

http://hdl.handle.net/10138/318221

https://doi.org/10.1016/j.ejps.2019.104991

cc_by_nc_nd

acceptedVersion

Downloaded from Helda, University of Helsinki institutional repository.

This is an electronic reprint of the original article.

This reprint may differ from the original in pagination and typographic detail.

Please cite the original version. 


\title{
Comparison of liquid chromatography-mass spectrometry and direct infusion microchip electrospray ionization mass spectrometry in global metabolomics of cell samples
}

Päivi Pöhö ${ }^{1}$, Katriina Lipponen ${ }^{1}$, Maxim M. Bespalov², Tiina Sikanen ${ }^{1}$, Tapio Kotiaho ${ }^{1,3}$, Risto Kostiainen $^{1 *}$

${ }^{1}$ Drug Research Program and Division of Pharmaceutical Chemistry and Technology, Faculty of Pharmacy, University of Helsinki, FI-00014 Helsinki, FINLAND

${ }^{2}$ Biomedicum Stem Cell Center, Stem Cells and Metabolism Research Program, Faculty of Medicine, University of Helsinki, FI-00014 Helsinki, FINLAND

${ }^{3}$ Department of Chemistry, Faculty of Science, University of Helsinki, FI-00014 Helsinki, FINLAND

* Corresponding author: E-mail: risto.kostiainen@,helsinki.fi. Phone: +358-2941 59134.

\begin{abstract}
In this study, the feasibility of direct infusion electrospray ionization microchip mass spectrometry (chip-MS) was compared to the commonly used liquid chromatography-mass spectrometry (LC-MS) in non-targeted metabolomics analysis of human foreskin fibroblasts (HFF) and human induced pluripotent stem cells (hiPSC) reprogrammed from HFF. The total number of the detected features with chip-MS and LC-MS were 619 and 1959, respectively. Approximately 25\% of detected features showed statistically significant changes between the cell lines with both analytical methods. The results show that chip-MS is a rapid and simple method that allows high sample throughput from small sample volumes and can detect the main metabolites and classify cells based on their metabolic profiles. However, the selectivity of chip-MS is limited compared to LC-MS and chip-MS may suffer from ion suppression.
\end{abstract}




\section{Keywords}

Metabolomics; electrospray; microchip; liquid chromatography-mass spectrometry; cells; direct infusion mass spectrometry.

\section{Introduction}

Metabolomics is a discipline that aims to identify and comprehensively analyze the changes of low-molecular weight metabolites in biological systems. These metabolites represent a diverse group of molecules that act as building blocks for several biological components or have otherwise essential roles in various cellular functions, such as regulation of enzyme expression and cell signaling [1-3]. Changes in metabolite levels are good measures of cell function, as they represent the downstream amplification of changes occurring in mRNA levels or proteome. Metabolomics is widely applied for biomarker discovery in disease diagnostics and prognosis, for understanding the mechanisms of disease evolution, and for identifying novel drug targets [1-3]. In stem cell research, detection of key metabolites at different cell differentiation states is particularly important to increase our understanding of the metabolic pathways essential for cell differentiation and maturation [4]. Stem cell metabolomics provides a valuable tool to study stem cell differentiation and reprogramming mechanisms [5-8] and to develop cell-based therapeutics $[9,10]$.

The sample pretreatment, separation, and detection method highly influences the range of compounds that can be detected. Currently, the most applied method in metabolomics research is mass spectrometry (MS) commonly coupled with a separation method such as liquid chromatography (LC) $[11,12]$, gas chromatography $(\mathrm{GC})[12,13]$, or capillary electrophoresis $(\mathrm{CE})[14,15]$. Alternatively, MS-based metabolomics can be conducted without the separation step using direct infusion MS [16, 17]. None of the existing methods can analyze all metabolites simultaneously. GC-MS is limited to 
volatile or semivolatile compounds and CE-MS mostly to ionic compounds. While LC-MS is a more universal method than GC-MS and CE-MS, selection of the LC method and the MS ionization technique limits the range of metabolites that can be detected. The advantages of LC-, GC-, or CE-MS are high selectivity and sensitivity. However, the analysis times of these techniques are substantially longer than that of direct infusion MS.

Direct infusion MS, including nanospray and microchip ionization techniques, allows rapid and straightforward analysis of a wide range of metabolites at high speed $[17,18]$. On the other hand, direct infusion MS experiments may suffer from matrix effects, which can cause ion suppression [19] and reduce sensitivity. The selectivity in direct infusion MS is also limited, as isobaric compounds cannot be separated based on the mass-to-charge ratio alone. Thus far, the applications of direct infusion MS in metabolomics have mainly focused on the analysis of lipids in serum and plasma or less frequently in other biological matrices such as tissue samples. Direct infusion MS methods applied for nontargeted metabolomics have been summarized in recent review articles $[16,17,20]$.

Microfabrication technology in particular provides an attractive avenue towards the development of rapid and highly automated microfluidic sample introduction platforms for MS. By permitting MS analysis of minimal sample amounts, the microchip technology can be considered as a prospective technique for improving the sensitivity and cost-efficiency of metabolomics research $[18,21]$. Thus far, a range of different on-chip ionization strategies have been proposed for coupling microfluidic devices with MS, mostly via electrospray ionization (ESI)[18, 22-24]. The possibility to integrate the on-chip ion source with another "upstream" microfluidic module, such as on-chip cell culture, cell lysis unit, and sample preparation further increases the appeal of microchip technology in metabolomics research. 
In this study, we examined the feasibility of microchip direct infusion MS for global metabolomics analysis in comparison with established LC-MS technique. The previously developed SU-8-based ESI microchip [25] was chosen as the representative microfluidic sample introduction platform and was connected to a high-resolution Orbitrap MS (HRMS) for rapid non-targeted metabolomics (later referred to as chip-MS). Reversed-phase (RP) C18 ultra-high performance liquid chromatography (UHPLC) coupled with electrospray quadrupole-time-of-flight mass spectrometry (ESI-QTOF-MS) was chosen as the gold standard reference method, as this method is one of the most commonly applied methods in non-targeted metabolomics (later referred to as LC-MS) [11]. A comparison between the two techniques was performed by analyzing human foreskin fibroblasts (HFF) and human-induced pluripotent stem cells (hiPSC) reprogrammed from HFF by both methods in positive ion mode. Thus far the differences in the performance of direct infusion MS and LC-MS in non-targeted metabolomics have been examined in only a few studies [26, 27]; only one study presented the output of the metabolomics comparison [26].

\section{Experimental section}

\subsection{Chemicals}

All aqueous solutions were prepared using ultrapure water purified with a MilliQ-system (Merck Millipore, France). LC-MS Chromasolv ${ }^{\circledR}$ graded methanol and isopropanol were from Sigma-Aldrich (Germany). Formic acid was from Merck (Germany). The internal standards verapamil and propranolol were from Sigma-Aldrich.

E8 media, Trypan Blue exclusion on Countess, non-essential amino acids, and penicillin-streptomycin mixture (Life Technologies, USA), TrypLE select and Glutamax (Gibco, USA), growth factor reduced Matrigel (Becton Dickinson, USA), fetal bovine serum Hyclone (GE Healthcare Life Sciences, USA), 
phosphate buffered saline (PBS), and Dulbecco's modified Eagle's medium (Sigma-Aldrich) were used for cell culturing and harvesting.

\subsection{Cell samples}

hiPSC cell line HEL24.3 was differentiated from HFF (ATCC) using non-integrative Sendai virusmediated transduction with Oct4, Sox2, Klf4, and c-Myc [28]. HEL24.3 were grown in E8 media on Matrigel-coated Petri dishes $(\varnothing 100 \mathrm{~mm})$ to near confluency in $5 \% \mathrm{CO}_{2}$ at $37^{\circ} \mathrm{C}$. Cells from six plates were then washed with PBS and treated with TrypLE select for $1-3$ min at $37^{\circ} \mathrm{C}$ and washed with cold PBS while still on the plate. The cells were next dissociated from the matrix by extensive tituration in $10 \mathrm{~mL}$ cold PBS and counted using Trypan Blue exclusion on Countess followed by centrifugation (200 x g, $5 \mathrm{~min}$ ) at room temperature. Pellets were resuspended in PBS. Five million cells derived from each plate were then transferred into silanized Eppendorf tube inserts and centrifuged ( $200 \mathrm{x} \mathrm{g}$, $3 \mathrm{~min}$ ). Excess of PBS was removed by suction and tubes were snap frozen in liquid nitrogen.

HFF were grown with Dulbecco's modified Eagle's medium on six Matrigel-coated Petri dishes $(\varnothing 100$ $\mathrm{mm})$ supplemented with fetal bovine serum, non-essential amino acids, penicillin-streptomycin mixture, and Glutamax. Four hours before cell harvesting, the cells were washed three times with PBS and media was changed to E8 to reduce the base media effect on metabolite detection. The cells were harvested exactly as hiPSC except that TrypLE treatment was 10 min and one million cells were placed into silanized tubes.

The blank samples were Matrigel-coated Petri dishes incubated with E8 media for four hours in a cell incubator, washed with PBS three times, and incubated with $1 \mathrm{ml}$ of PBS at room temperature for an additional 10 min. PBS aliquots collected from six plates were subsequently used as blank samples. All samples were stored at $-80^{\circ} \mathrm{C}$ prior to sample preparation. 


\subsection{Sample preparation}

Cells were lysed by adding $50 \mu \mathrm{L}$ of MilliQ water to frozen cell pellets and sonicating them in icecold ultrasound bath for 15 min two times with mixing between cycles. Next, $20 \mu \mathrm{L}$ of the cell lysate was transferred to a new silanized insert followed by addition of $10 \mu \mathrm{L}$ of internal standard solution (4 $\mu \mathrm{g} \mathrm{mL}^{-1}$ of verapamil and propranolol in methanol) and $100 \mu \mathrm{L}$ of methanol. The cell extracts were then vortexed and incubated on ice for $20 \mathrm{~min}$. After incubation, the extracts were centrifuged (7378 $\mathrm{x} \mathrm{g}, 10 \mathrm{~min}$ ) at room temperature and the supernatant was transferred into a vial with a new silanized insert and stored at $-20^{\circ} \mathrm{C}$ until analysis. For LC-MS, the samples were injected as such. For chip-MS, 10\% FA in MilliQ was added to the samples to achieve a final solvent composition of 70:29:1 (MeOH: $\left.\mathrm{H}_{2} \mathrm{O}: \mathrm{HCOOH}\right)$.

\subsection{Chip-MS analysis}

Free standing, three-layered direct infusion ESI microchips were fabricated from SU-8 by UV lithography and release etching as described previously [29]. Before use, polydimethylsiloxane sheets with 2-mm inlet holes were attached on top of the sample inlet to avoid liquid spreading onto the relatively hydrophilic SU-8 surface (around the inlet). In this manner, the applied sample volume could be increased to $5 \mu \mathrm{L}$ to ensure that the duration of sample infusion was long enough for collection of proper MS spectra. For analysis of the cell extracts, the chips were coupled to a Q-Exactive Hybrid Quadrupole Orbitrap Mass Spectrometer controlled via Xcalibur software (Thermo Fisher Scientific, USA). The MS data were collected in positive ion profile mode with ion transfer tube temperature set at $200^{\circ} \mathrm{C}, \mathrm{m} / \mathrm{z}$ range of $100-1500$, and scan frequency of $3 \mathrm{~Hz}$. The MS resolution was $70000 \mathrm{FWHM}$ at $m / z$ 200. A custom-made high voltage supply was used for application of the ESI voltage ( $2.5 \mathrm{kV})$ via a platinum electrode inserted into the sample inlet. The spectrum was recorded for $0.3 \mathrm{~min}$. Each 
sample was analyzed in triplicate with careful rinsing of the channel with a $\mathrm{MeOH}: \mathrm{H}_{2} \mathrm{O}(1: 1)$ between runs to avoid possible memory effect.

\subsection{LC-MS analysis}

LC-MS analysis was performed with a Xevo QTOF mass spectrometer combined with an Acquity UHPLC and controlled with MassLynx ${ }^{\mathrm{TM}} 4.1$ software (Waters, USA). Data were collected as centroid in positive ion mode, applying a mass range $\mathrm{m} / \mathrm{z} 100-1000$ with a scan time of $150 \mathrm{~ms}$ (extended dynamic range). Resolution was 8000 FWHM and lock mass correction of Leucine-Enkephaline was applied. The other MS operation parameters were the following: capillary voltage $2 \mathrm{kV}$, sampling cone voltage $20 \mathrm{~V}$, extraction cone voltage $1 \mathrm{~V}$, source temperature $150^{\circ} \mathrm{C}$, desolvation temperature $450^{\circ} \mathrm{C}$, desolvation gas flow $800 \mathrm{~L} \mathrm{~h}^{-1}$, and cone gas flow $20 \mathrm{~L} \mathrm{~h}^{-1}$.

The UHPLC RP column was a BEH C18 (2.1 mm x $100 \mathrm{~mm}, 1.7 \mu \mathrm{m}$, Waters). Eluent A was 0.1\% formic acid in MilliQ water and eluent B was $0.1 \%$ formic acid in methanol. A linear gradient started from $5 \% \mathrm{~B}$ and proceeded to $100 \% \mathrm{~B}$ in $15 \mathrm{~min}$ and was maintained for $10 \mathrm{~min}$ followed by the reequilibration step for $5 \mathrm{~min}$. The total runtime was $30 \mathrm{~min}$ with a flow rate of $0.3 \mathrm{~mL} \mathrm{~min}{ }^{-1}$. An autosampler was cooled to $10^{\circ} \mathrm{C}$ and the column thermostated for $25^{\circ} \mathrm{C}$ degrees. The injection volume was $5 \mu \mathrm{L}$.

\subsection{Data preprocessing and analysis}

Data preprocessing of both chip-MS and LC-MS datasets was performed with MzMine 2 [30]. Data preprocessing for LC-MS included following steps: mass detection with centroid algorithm, chromatogram builder, deconvolution with local minimum search, isotopic peaks grouper, alignment with join aligner, filtering, gap filling with peak finder, and adduct search. For chip-MS data 
preprocessing steps were: mass detection with exact mass filter, chromatogram builder, isotopic peaks grouper, alignment with join aligner, filtering, gap filling with same RT and m/z range cap filler, and adduct search. Detailed data preprocessing parameters are presented in the Supplementary data (Table S1) and descriptions of preprocessing steps and applied algorithms are available at website: http://mzmine.github.io/ . Prior to preprocessing, LC-MS raw data files were converted to netCDF files with DataBridge (Waters). Relative normalization of peak abundances (chip-MS) and extracted ion chromatogram peak heights (LC-MS) against those of the internal standard (verapamil, $\mathrm{m} / \mathrm{z} 455.29$ ) were applied for both datasets. For chip-MS, the average values of normalized peak abundances of three replicate measurements were calculated, one outlier sample was removed, and an average value was further applied in the data analysis. Identification of detected compounds was based on the search with accurate mass ( $3 \mathrm{ppm}$ ) from the human metabolome database (HMDB) and Lipid Maps.

Data analyses were performed with $\mathrm{R}$ version x64 3.0.2. utilizing metadar-package [31]. Zero values in the data were imputed with half of the minimum algorithm, replacing zero values with half of the minimum value of the corresponding feature across the samples. Principal component analysis (PCA) was performed after centering and $\log 2$ transformation. Unpaired $t$-test with false discovery rate correction (FDR, Benjamini-Hochberg) was applied and fold changes were calculated.

\section{Results and discussion}

\subsection{Comparison of LC-MS and chip-MS}

The main anticipated advantage of chip-MS over LC-MS in metabolomics is its feasibility for rapid and straightforward analysis. A run time in chip-MS was $0.3 \mathrm{~min}$, while a run time in LC-MS was 30 min. High sample throughput is of utmost importance when analyzing a large sample series as 
commonly encountered in clinical settings. Although sample preparation is the most time-consuming step for both methods, it can be automated and performed in batch mode.

As the data produced by chip-MS and LC-MS are different, it is impossible to use exactly the same data preprocessing tools and steps. However, by applying the same software (MzMine 2) the data preprocessing was performed as similarly as possible (Table S1). In principal, data preprocessing is easier and faster in direct infusion, where only mass spectra (Fig. 1B and 1D) are combined through all data points and the combined spectra are aligned across the samples. In LC-MS, the features are selected from a chromatogram (Fig. 1A and 1C) with peak deconvolution algorithms, which requires additional preprocessing steps. This retention time alignment might also cause bias due to possible retention time drifts, especially in the analysis of large metabolomics sample sets.

A wide range of metabolites from polar small molecules to lipids were detected with both the chipMS and the LC-MS methods (Fig. 1). The main metabolites identified were amino acids, amino acid derivatives, carnitines, nucleotides, sugars, sugar phosphates, fatty acids, fatty acid and steroid derivatives, and a high number of lipids from classes such as monoglycerides (MG), phosphatidylcholines (PC), phosphatidylethanolamines (PE), and sphingomyelins (SM). In addition, several other metabolites that include oxygen as a heteroatom in aliphatic structures (such as alcohols, esters, and carboxylic acids) were found based on the accurate mass measurements. The metabolites were detected as their protonated molecules or sodium or potassium adduct ions (or combinations thereof). Adduct ions were observed as the cell samples contained residual sodium and potassium salts after extraction. In C18 reversed-phase liquid chromatography, the salt concentration in ESI was low as salts were eluted at void volume. Salts were therefore separated from most of the metabolites, resulting in decreased adduct ion intensities and formation of mainly protonated molecules. In chipMS, significantly more abundant adduct ions were detected, since the residual salts were not chromatographically separated from metabolites and the relatively high salt concentration in the ESI 
favored adduct ion formation. However, some of the most polar metabolites (such as sugars and their derivatives, amino acids, and nucleotides) were eluted in the LC-MS in the void volume together with the residual salts, resulting in formation of abundant adduct ions similar to the chip-MS.

The total number of the detected features with chip-MS and LC-MS were 619 and 1959, respectively. The number of detected features is largely dependent on the peak capacity and sensitivity of the analytical method. The peak capacity of Orbitrap MS (resolution 70000 FWHM) can be estimated to be about seven-fold higher than that of QTOF-MS (resolution 8000 FWHM) applied in this work. It is worth noting that the resolution with Orbitrap MS was 70000 at $\mathrm{m} / \mathrm{z} 200$, but only about 30000 at $\mathrm{m} / \mathrm{z}$ 1000 as the resolution in Orbitrap MS is mass range dependent [32]. As the peak capacity for UHPLC in our experiments with a 15 -min gradient time is estimated to be about 380 according to the literature $[33,34]$, the overall peak capacity in the LC-MS analysis is about 50 times higher than that obtained with the chip-MS. The lower peak capacity with the chip-MS results in an increased number of overlapping features, particularly with lipids. For example, in the LC-MS data of hiPSC and HFF, the extracted ion chromatograms of $m / z 806.57(\Delta m / z$ 0.04) showed several peaks, whereas the chip-MS spectrum showed only one peak between $\mathrm{m} / \mathrm{z}$ 806.5-806.6 and the differences of specific lipid species between hiPSC and HFF cell types were not observed (Fig. 2). A closer examination of spectra of the peaks detected in the extracted ion LC-MS chromatogram of $m / z 806.57$ at retention times of 17.53 and $17.33 \mathrm{~min}$ revealed that these most likely represent isomers of $[\mathrm{PC}(38: 6)+\mathrm{H}]^{+}($exact mass $\mathrm{m} / \mathrm{z}$ 806.5694, $\mathrm{C}_{46} \mathrm{H}_{80} \mathrm{NO}_{8} \mathrm{P}+\mathrm{H}$ ), and the peaks at 17.88, 18.0, and 18.19 min represent isomers of $[\mathrm{PC}(36: 3)+\mathrm{Na}]^{+}$(exact mass $m / z$ 806.5670, $\mathrm{C}_{44} \mathrm{H}_{82} \mathrm{NO}_{8} \mathrm{P}+\mathrm{Na}$ ) (Fig. 2). The mass difference between the ions $[\mathrm{PC}(38: 6)+\mathrm{H}]^{+}$and $[\mathrm{PC}(36: 3)+\mathrm{Na}]^{+}$is only $2.4 \mathrm{mDa}$, which suggests that mass resolution over about 350000 would have been required to distinguish these ions in the chip-MS spectra. Although such high resolution is not achievable with the Orbitrap used in our chip-MS experiments, this is possible with higher mass resolution mass spectrometers such as next-generation Orbitraps or Fourier 
transform ion cyclotron mass spectrometers. In addition to the example above, the [M+2] isotopes of lipids may overlap with lipids belonging to the same lipid class but with one less double bond (Fig. 2). These types of overlaps may especially hinder the detection of differences of less abundant metabolites between samples. These examples clearly show that the use of LC-MS, even with a moderate mass resolution, provides significantly better selectivity and thus more detailed information in non-targeted metabolomics than direct MS, even in combination with modern ultra-high mass resolution mass spectrometers. However, this comes at the cost of analysis time.

Another reason for the lower number of detected features with chip-MS is ion suppression. This is more evident in chip-MS than in LC-MS. In addition to salts, some phospholipids such as phosphatidylcholines (which are easily ionized in ESI) may suppress ionization of metabolites that have lower ionization efficiency [35, 36]. For example, the intensities of several lysolipids were below the detection threshold in chip-MS but were clearly detected with LC-MS. On the other hand, salts may assist ionization of some low proton affinity metabolites, which are poorly ionized via a proton transfer reaction but are ionized more efficiently via adduct ion formation $[37,38]$. As salts were separated in the LC-MS but not in the chip-MS, some metabolites (including low proton affinity compounds in compound classes; carboxylic acids, amino acids, carnitines, sugar derivatives, sugar phosphates, phosphates, and nucleosides) were detected only with chip-MS as their adduct ions but were not detected in LC-MS.

To determine the feasibility of chip-MS and LC-MS in metabolomics studies, the repeatability of the methods was studied by adding the internal standards propranolol and verapamil to the cell samples. The relative standard deviations (RSD\%) of ion intensities measured using LC-MS for propranolol and verapamil in HFF samples were $6.0 \%$ and $5.1 \%$ and in hiPSC samples $6.1 \%$ and $4.2 \%$, respectively. The RSD\% measured using chip-MS for propranolol and verapamil in HFF samples were $8.6 \%$ and $8.6 \%$ and in hiPSC samples $10.6 \%$ and $6.4 \%$, respectively. These results indicate that the 
repeatability within each cell sample was good with both methods. Our experiments also show that the signal intensities of the internal standards were about two times lower in hiPSC samples than in HFF samples in the chip-MS measurements, but no significant differences were observed in the LC-MS measurements (Fig. 3). In our experiments, the biomass of the cells was the same in all samples. However, the number of cells was higher in hiPSC samples (5 million) than in HFF samples (1 million), as hiPSC are significantly smaller than HFF. Consequently, the total surface area (i.e. the total amount of cell membrane and thus phospholipid concentration) is higher in hiPSC than in HFF samples. Certain phospholipids, such as phosphatidylcholines, are known to cause ion suppression in ESI $[35,36]$. Accordingly, it is possible that ion suppression is stronger with hiPSC than with HFF samples when measured by chip-MS, which is more prone to ion suppression than LC-MS. These results show that normalization using internal standards is necessary for comparison of metabolic changes between cell samples when using the chip-MS approach.

\subsection{Data-analysis of cell samples}

An unsupervised PCA was used to study differences between HFF and hiPSC datasets. PCA showed clear grouping of the cell types, indicating differences in the metabolic profiles of HFF and hiPSC with both analytical methods (Fig. 4). This observation demonstrates that the metabolic profiles from the different cell types can be separated with chip-MS and with LC-MS.

To determine statistically significant changed features between the HFF and hiPSC datasets, we used $t$-test with FDR corrected $p$-value $<0.01(q$-value $<0.01)$ and fold change of at least 2 for the datasets obtained with chip-MS and LC-MS. With these criteria, 435 features detected with the LC-MS were changed, of which 304 features were upregulated and 131 were downregulated in hiPSC compared to HFF. The number of changed features detected with chip-MS was 157, of which 88 features were upregulated and 69 were downregulated in hiPSC. These results show that only about one third of the 
changed features detected with LC-MS can be detected with chip-MS. This is most likely due to the lower selectivity and higher ion suppression of chip-MS (see above). The number of significantly changed features $(q<0.01$, fold $>2)$ with both analytical methods (with a maximum mass difference of $20 \mathrm{ppm}$ ) was 44 . These metabolites were tentatively identified based on their accurate masses by applying the HMDB and are presented with fold changes (hiPSC/HFF) and $q$-values in Table 1 . The fold changes mainly correlated well between the analytical methods, although three phospholipids at $m / z 754.536,834.599$, and 818.603 were changed in the opposite direction. This may be due to peak overlap and domination of sodium adducts in chip-MS measurements. The most downregulated compounds were N-methylnicotinamide, creatine, L-carnitine, and PCs and PEs. The most clearly upregulated metabolites were MGs, aminoadipic acid, choline, niacinamide, and some PCs. These results suggest that the changes of the identified metabolites can be determined relatively reliably with fold changes over two.

\section{Conclusions}

In this study, we compared the feasibility of chip-MS and LC-MS for non-targeted metabolomics with HFF and hiPSC cell samples. We detected an approximately three-fold higher number of features and changed features between HFF and hiPSC cell samples with LC-MS than with chip-MS. This was due to the better selectivity and lower ion suppression of LC-MS. The chip-MS measurement favored the formation of adduct ions, since the salts were not separated from metabolites as in LC-MS. Adduct ion formation may complicate the interpretation of mass spectra and identification of metabolites. However, adduct ion formation can also increase the ionization efficiency of low proton affinity metabolites, which are poorly ionized by proton transfer reaction. Despite the challenges associated with selectivity and ion suppression in chip-MS, the two different cell groups could be classified and separated with PCA and several changed metabolites were identified. Furthermore, chip-MS is a 
simple, fast, and sensitive method for metabolome analysis in minimum sample volumes. Moreover, chip-MS can be easily automated for high sample throughput, as required for analysis of clinical samples.

\section{Acknowledgements}

We would like to thank Prof. Timo Otonkoski and the Biomedicum Stem Cell Center for providing the cell samples. Financial support was provided by the Business Finland Large Strategic Opening project (40395/13), the European Research Council (grant no. 311705, TS), and the University of Helsinki Doctoral Program in Drug Research.

\section{Conflict of interests}

The authors declare no competing financial interests.

\section{Appendix a. Supplementary data}

\section{References}

[1] G.J. Patti, O. Yanes, G. Siuzdak, Innovation: Metabolomics: the apogee of the omics trilogy, Nat. Rev. Mol. Cell Biol. 13 (2012) 263-269.

[2] D.S. Wishart, Emerging applications of metabolomics in drug discovery and precision medicine, Nat. Rev. Drug Discov. 15 (2016) 473-484.

[3] C.H. Johnson, J. Ivanisevic, G. Siuzdak, Metabolomics: Beyond biomarkers and towards mechanisms, Nat. Rev. Mol. Cell Biol. 17 (2016) 451-459. 
[4] J.A. Gaspar, M.X. Doss, J.G. Hengstler, C. Cadenas, J. Hescheler, A. Sachinidis, Unique metabolic features of stem cells, cardiomyocytes, and their progenitors, Circ. Res. 114 (2014) 13461360.

[5] O. Yanes, J. Clark, D.M. Wong, G.J. Patti, A. Sánchez-Ruiz, H.P. Benton, S.A. Trauger, C.

Desponts, S. Ding, G. Siuzdak, Metabolic oxidation regulates embryonic stem cell differentiation, Nat. Chem. Biol. 6 (2010) 411-417.

[6] A.D. Panopoulos, O. Yanes, S. Ruiz, Y.S. Kida, D. Diep, R. Tautenhahn, A. Herrerías, E.M. Batchelder, N. Plongthongkum, M. Lutz, W.T. Berggren, K. Zhang, R.M. Evans, G. Siuzdak, J.C.I. Belmonte, The metabolome of induced pluripotent stem cells reveals metabolic changes occurring in somatic cell reprogramming, Cell Res. 22 (2012) 168-177.

[7] J.K. Meissen, B.T.K. Yuen, T. Kind, J.W. Riggs, D.K. Barupal, P.S. Knoepfler, O. Fiehn, Induced Pluripotent Stem Cells Show Metabolomic Differences to Embryonic Stem Cells in Polyunsaturated Phosphatidylcholines and Primary Metabolism, PLoS One 7 (2012) e46770.

[8] B.A. Beyer, M. Fang, B. Sadrian, J.R. Montenegro-Burke, W.C. Plaisted, B.P.C. Kok, E. Saez, T. Kondo, G. Siuzdak, L.L. Lairson, Metabolomics-based discovery of a metabolite that enhances oligodendrocyte maturation, Nat. Chem. Biol. 14 (2018) 22-28.

[9] D. Srivastava, N. DeWitt, In Vivo Cellular Reprogramming: The Next Generation, Cell 166 (2016) 1386-1396.

[10] K. Liu, C. Yu, M. Xie, K. Li, S. Ding, Chemical Modulation of Cell Fate in Stem Cell Therapeutics and Regenerative Medicine, Cell Chem. Biol. 23 (2016) 893-916. 
[11] B. Madji Hounoum, H. Blasco, P. Emond, S. Mavel, Liquid chromatography-high-resolution mass spectrometry-based cell metabolomics: Experimental design, recommendations, and applications, TrAC, Trends Anal. Chem. 75 (2016) 118-128.

[12] S. Naz, D. C. Moreira Dos Santos, A. García, C. Barbas, Analytical protocols based on LC-MS, GC-MS and CE-MS for nontargeted metabolomics of biological tissues, Bioanalysis 6 (2014) 16571677.

[13] D.J. Beale, F.R. Pinu, K.A. Kouremenos, M.M. Poojary, V.K. Narayana, B.A. Boughton, K. Kanojia, S. Dayalan, O.A.H. Jones, D.A. Dias, Review of recent developments in GC-MS approaches to metabolomics-based research, Metabolomics 14 (2018) 152.

[14] R. Ramautar, G.W. Somsen, G.J. de Jong, CE-MS for metabolomics: Developments and applications in the period 2014-2016, Electrophoresis 38 (2017) 190-202.

[15] A. Hirayama, M. Wakayama, T. Soga, Metabolome analysis based on capillary electrophoresismass spectrometry, TrAC, Trends Anal. Chem. 61 (2014) 215-222.

[16] R. González-Domínguez, A. Sayago, Fernández-Recamales, Direct infusion mass spectrometry for metabolomic phenotyping of diseases, Bioanalysis 9 (2017) 131-148.

[17] J. Draper, A.J. Lloyd, R. Goodacre, M. Beckmann, Flow infusion electrospray ionisation mass spectrometry for high throughput, non-targeted metabolite fingerprinting: A review, Metabolomics 9 (2013) 4-29.

[18] T. Sikanen, S. Franssila, T.J. Kauppila, R. Kostiainen, T. Kotiaho, R.A. Ketola, Microchip technology in mass spectrometry, Mass Spectrom. Rev. 29 (2010) 351-391. 
[19] T.M. Annesley, Ion suppression in mass spectrometry, Clin. Chem. 49 (2003) 1041-1044.

[20] M. Wang, C. Wang, R.H. Han, X. Han, Novel advances in shotgun lipidomics for biology and medicine, Prog. Lipid Res. 61 (2016) 83-108.

[21] M. de Raad, C.R. Fischer, T.R. Northen, High-throughput platforms for metabolomics, Curr. Opin. Chem. Biol. 30 (2016) 7-13.

[22] X. He, Q. Chen, Y. Zhang, J.-M. Lin, Recent advances in microchip-mass spectrometry for biological analysis, TrAC, Trends Anal. Chem. 53 (2014) 84-97.

[23] X. Feng, B.-F. Liu, J. Li, X. Liu, Advances in coupling microfluidic chips to mass spectrometry, Mass Spectrom. Rev. 34 (2015) 535-557.

[24] X. Wang, L. Yi, N. Mukhitov, A.M. Schrell, R. Dhumpa, M.G. Roper, Microfluidics-to-mass spectrometry: A review of coupling methods and applications, J. Chromatogr. A 1382 (2015) 98116.

[25] T. Sikanen, S. Tuomikoski, R.A. Ketola, R. Kostiainen, S. Franssila, T. Kotiaho, Analytical characterization of microfabricated SU-8 emitters for electrospray ionization mass spectrometry, J. Mass Spectrom. 43 (2008) 726-735.

[26] L. Lin, Q. Yu, X. Yan, W. Hang, J. Zheng, J. Xing, B. Huang, Direct infusion mass spectrometry or liquid chromatography mass spectrometry for human metabonomics? A serum metabonomic study of kidney cancer, Analyst 135 (2010) 2970-2978. 
[27] M. Lísa, E. Cífková, M. Khalikova, M. Ovčačíková, M. Holčapek, Lipidomic analysis of biological samples: Comparison of liquid chromatography, supercritical fluid chromatography and direct infusion mass spectrometry methods, J. Chromatogr. A 1525 (2017) 96-108.

[28] R. Trokovic, J. Weltner, T. Otonkoski, Generation of iPSC line HEL24.3 from human neonatal foreskin fibroblasts, Stem Cell Res. 15 (2015) 266-268.

[29] S. Tuomikoski, T. Sikanen, R.A. Ketola, R. Kostiainen, T. Kotiaho, S. Franssila, Fabrication of enclosed SU-8 tips for electrospray ionization-mass spectrometry, Electrophoresis 26 (2005) 46914702.

[30] T. Pluskal, S. Castillo, A. Villar-Briones, M. Orešič, MZmine 2: Modular framework for processing, visualizing, and analyzing mass spectrometry-based molecular profile data, BMC Bioinform. 11 (2010) 395.

[31] G. Peddinti, Metadar; R package for metabolomics data analysis. https://code.google.com/archive/p/metadar. Updated 2013.

[32] A. Makarov, E. Denisov, O. Lange, Performance Evaluation of a High-field Orbitrap Mass Analyzer, J. Am. Soc. Mass Spectrom. 20 (2009) 1391-1396.

[33] I.D. Wilson, J.K. Nicholson, J. Castro-Perez, J.H. Granger, K.A. Johnson, B.W. Smith, R.S. Plumb, High Resolution "Ultra Performance" Liquid Chromatography Coupled to oa-TOF Mass Spectrometry as a Tool for Differential Metabolic Pathway Profiling in Functional Genomic Studies, J. Proteome Res. 4 (2005) 591-598. 
[34] I.D. Wilson, R. Plumb, J. Granger, H. Major, R. Williams, E.M. Lenz, HPLC-MS-based methods for the study of metabonomics, J. Chromatogr. B Anal. Technol. Biomed. Life Sci. 817 (2005) 67-76.

[35] C. Ferreiro-Vera, F. Priego-Capote, Luque de Castro, M. D., Comparison of sample preparation approaches for phospholipids profiling in human serum by liquid chromatography-tandem mass spectrometry, J. Chromatogr. A 1240 (2012) 21-28.

[36] S. Tulipani, R. Llorach, M. Urpi-Sarda, C. Andres-Lacueva, Comparative analysis of sample preparation methods to handle the complexity of the blood fluid metabolome: When less is more, Anal. Chem. 85 (2013) 341-348.

[37] J.A. Bowden, C.J. Albert, O.S. Barnaby, D.A. Ford, Analysis of cholesteryl esters and diacylglycerols using lithiated adducts and electrospray ionization-tandem mass spectrometry, Anal. Biochem. 417 (2011) 202-210.

[38] K.L. Duffin, J.D. Henion, J.J. Shieh, Electrospray and Tandem Mass Spectrometry Characterization of Acylglycerol Mixtures That Are Dissolved in Nonpolar Solvents, Anal. Chem. 63 (1991) 1781-1788 


\section{LC-MS}
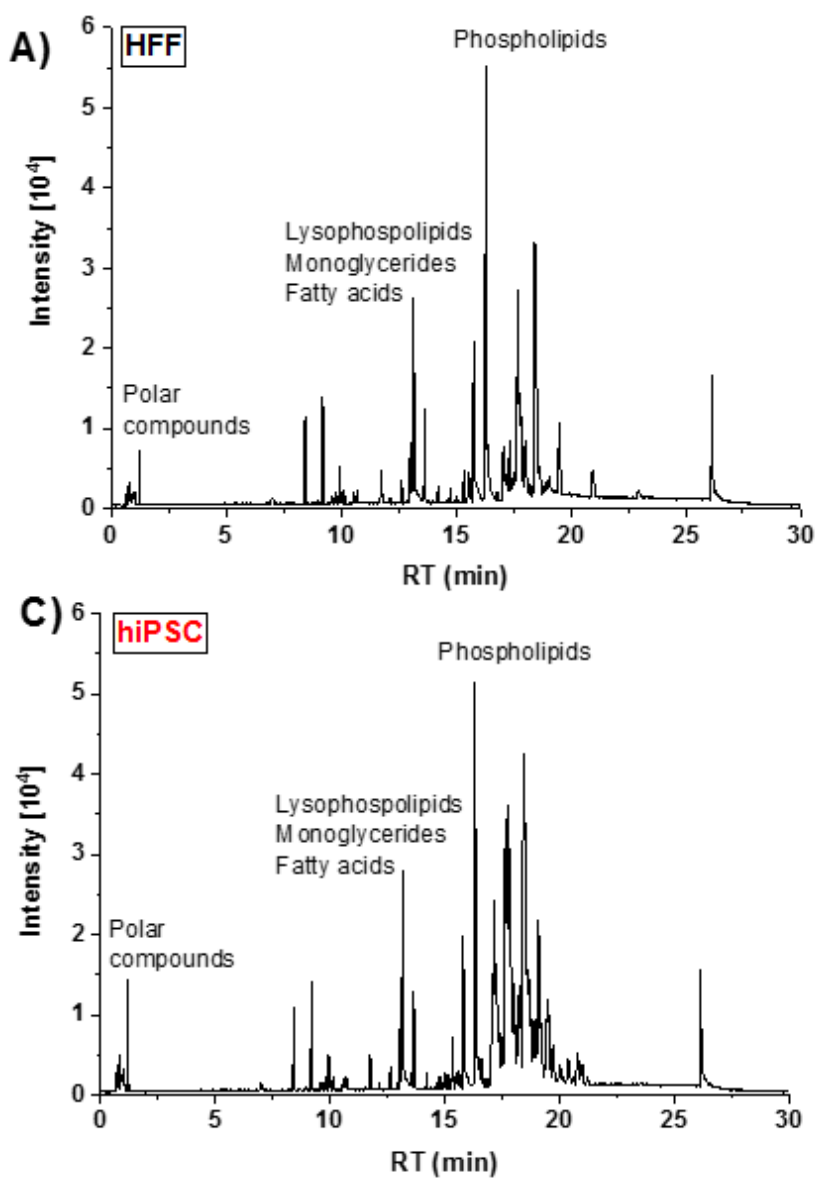

Chip-MS
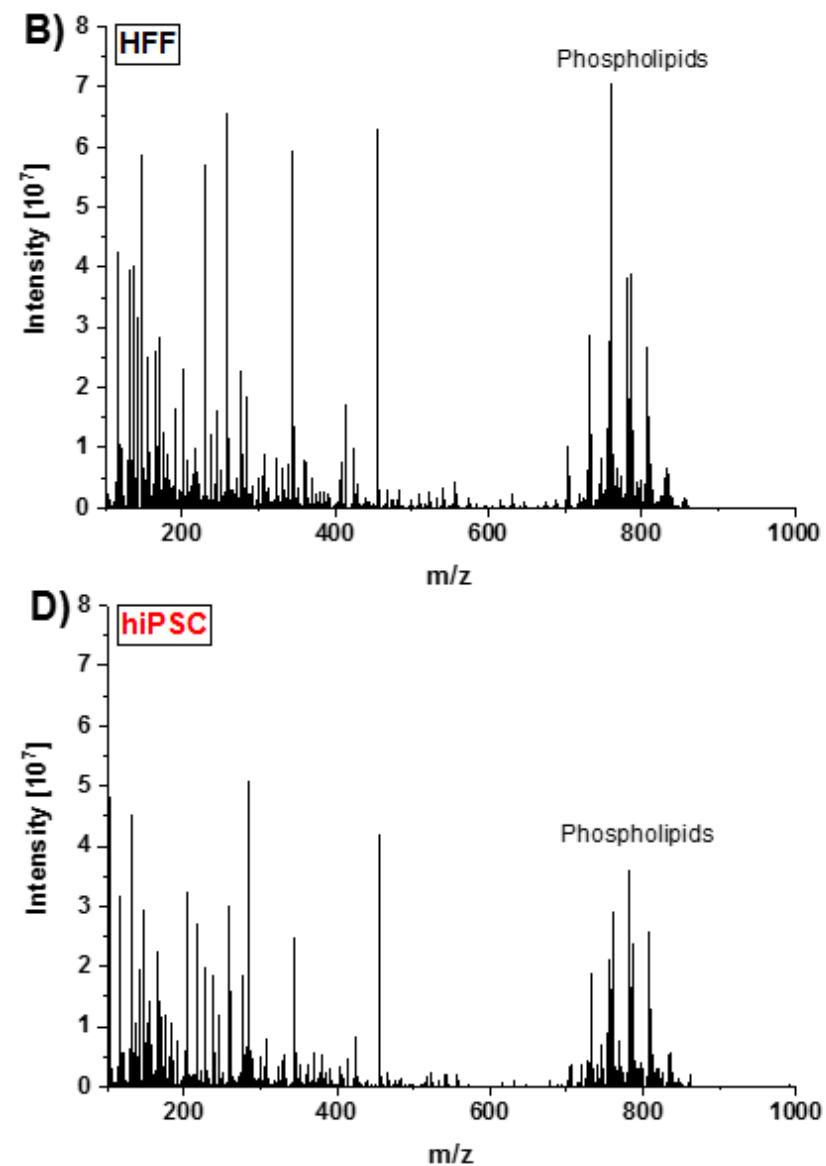

Fig. 1. Base peak chromatograms of HFF and hiPSC samples (A and C) with reversed phase UHPLCESI-QTOF-MS with mass range $100-1000 \mathrm{~m} / \mathrm{z}$. Combined spectra of HFF and hiPSC samples $(0.3$ min) with ESI-microchip-Orbitrap-MS using resolution of 70000 FWHM (B and D). 

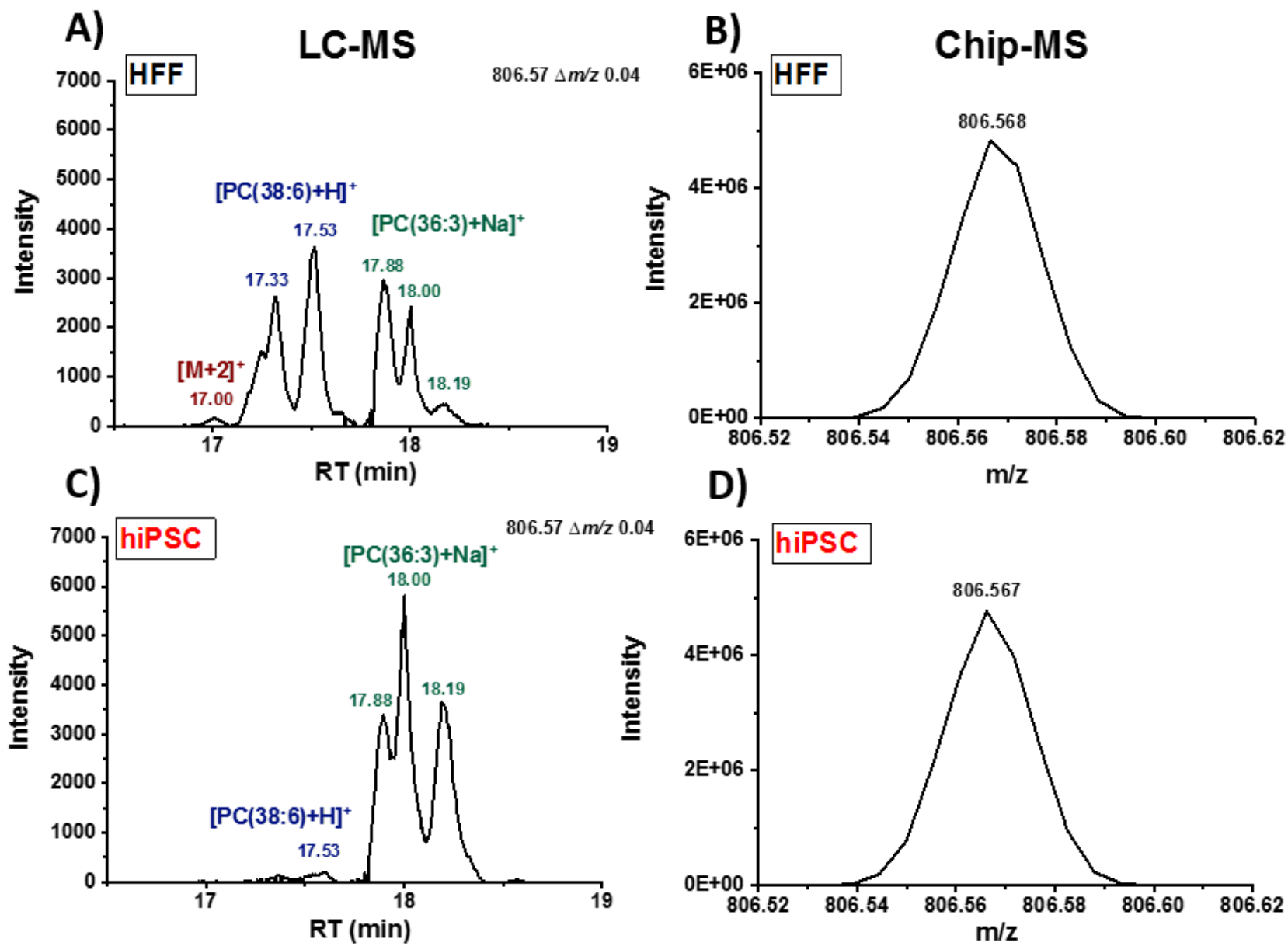

Fig. 2. UHPLC-ESI-QTOF-MS ion chromatograms of features detected at $m / z \quad 806.57(\Delta m / z \quad 0.04)$ from HFF and hiPSC samples (A and C). The peaks in the chromatograms correspond the protonated phosphatidylcholine $[\mathrm{PC}(38: 6)+\mathrm{H}]^{+}$(blue), phosphatidylcholine sodium adduct $[\mathrm{PC}(36: 3)+\mathrm{Na}]^{+}$ (green), and (M+2) isotope of m/z $804.55[\mathrm{PC}(38: 7)+\mathrm{H}]^{+}$. ESI-microchip-Orbitrap-HRMS spectra of HFF and hiPSC samples with mass window of $m / z 806.57 \pm 0.05$ (B and D). 

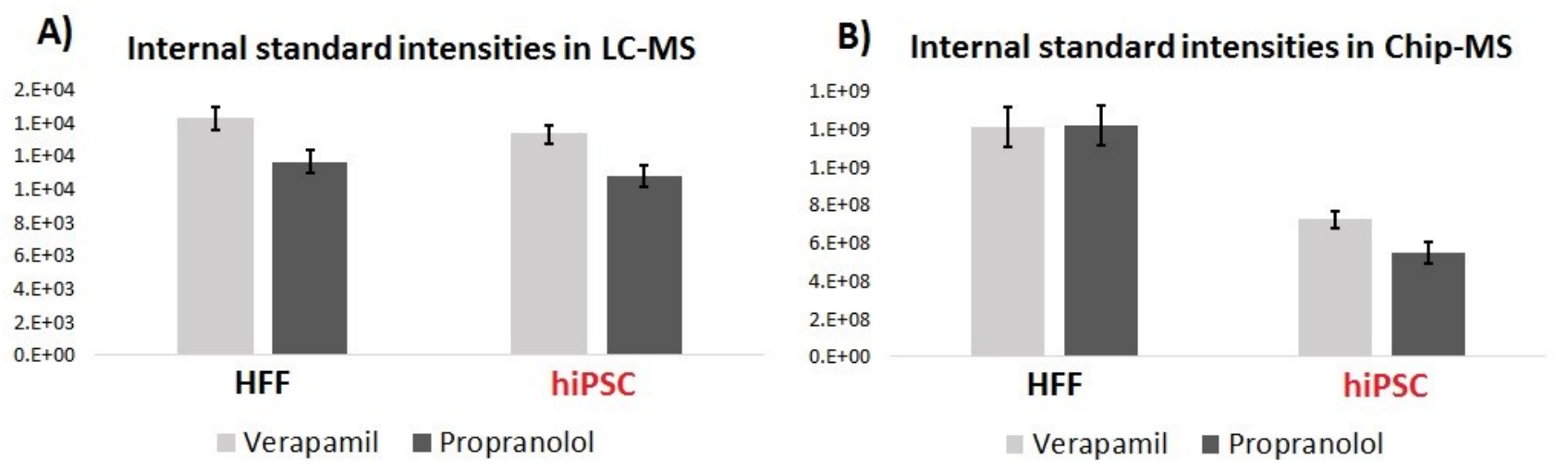

Fig. 3. Intensity levels of internal standards verapamil and propranolol in HFF and hiPSC samples in UHPLC-ESI-QTOF-MS (A) and ESI-microchip-Orbitrap-HRMS (B) analysis.
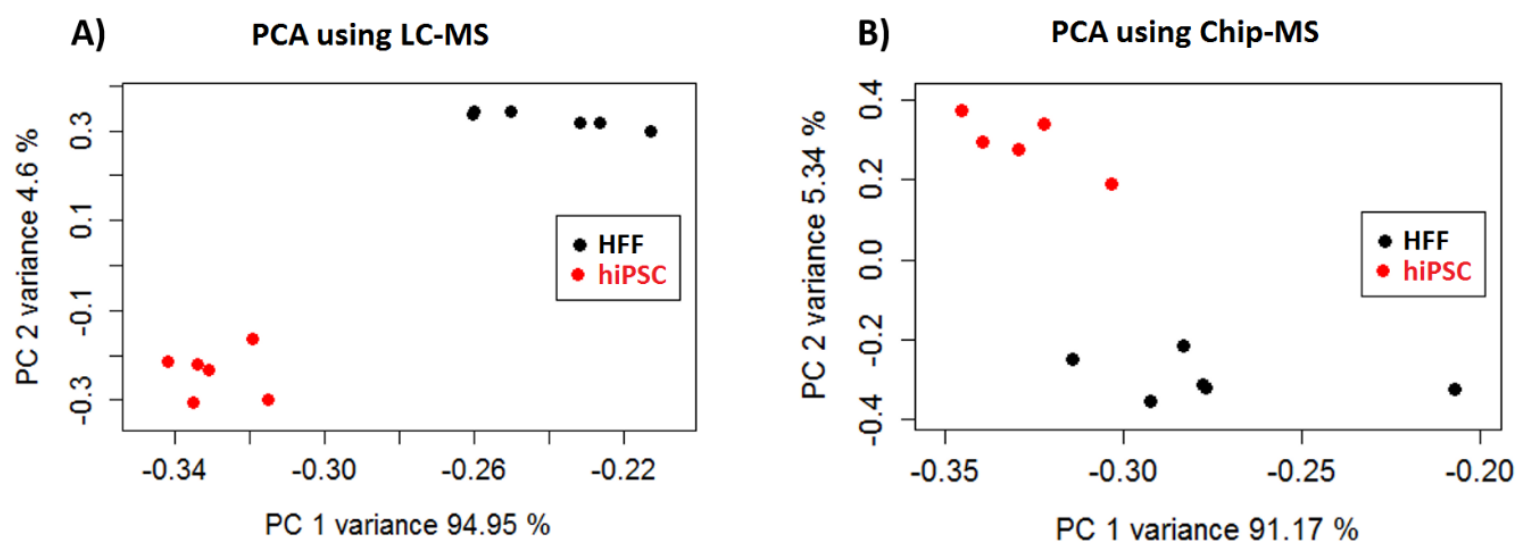

Fig. 4. Principal component analysis (PCA) score plots of normalized and scaled intensities in HFF (black) and hiPSC (red) samples using UHPLC-ESI-QTOF-MS (A) and ESI-microchip-OrbitrapHRMS (B).

Table 1. Changed metabolites between cell samples detected with both methods (Fold $>2, q<0.01$ ). Red indicates upregulated and blue indicates downregulated metabolites in hiPSC compared to HFF. 


\begin{tabular}{|c|c|c|c|c|c|c|c|}
\hline \multicolumn{3}{|c|}{$\begin{array}{c}\text { LC-MS } \\
\text { Log } 2 \\
\text { q-value } \\
\text { hiPSC/ } \mathrm{m} / \mathrm{z} \\
\text { HFF }\end{array}$} & \multicolumn{3}{|c|}{$\begin{array}{c}\text { Chip-MS } \\
\text { Log } 2 \\
\text { q-value hiPSC/ } \mathrm{m} / \mathrm{z} \\
\text { HFF }\end{array}$} & $\begin{array}{l}\text { Elemental } \\
\text { formula }\end{array}$ & $\begin{array}{l}\text { Tentative } \\
\text { identificatio }\end{array}$ \\
\hline $3.5 \mathrm{E}-05$ & 5 & \begin{tabular}{|l|}
379.2863 \\
\end{tabular} & $1.9 \mathrm{E}-04$ & 5 & \begin{tabular}{|l|}
379.2816 \\
\end{tabular} & $\mathrm{C} 21 \mathrm{H} 40 \mathrm{O} 4+\mathrm{Na}$ & MG (18:1) \\
\hline 4.6E-07 & 4 & \begin{tabular}{|l|}
162.0779 \\
\end{tabular} & $1.6 \mathrm{E}-04$ & 2 & 162.076 & $\mathrm{C} 6 \mathrm{H} 11 \mathrm{NO} 4+\mathrm{H}$ & Aminoadipic acid \\
\hline $9.5 \mathrm{E}-06$ & 3 & \begin{tabular}{|l|}
772.6278 \\
\end{tabular} & $1.4 \mathrm{E}-06$ & 4 & \begin{tabular}{|l|}
772.6197 \\
\end{tabular} & $\mathrm{C} 44 \mathrm{H} 86 \mathrm{NO} 07 \mathrm{P}+\mathrm{H}$ & $P C(36: 2 e)$ \\
\hline $1.3 \mathrm{E}-04$ & 3 & \begin{tabular}{|l|}
353.2673 \\
\end{tabular} & $1.2 \mathrm{E}-04$ & 5 & \begin{tabular}{|l|}
353.2659 \\
\end{tabular} & $\mathrm{C} 19 \mathrm{H} 38 \mathrm{O} 4+\mathrm{Na}$ & MG (16:0) \\
\hline $1.1 \mathrm{E}-11$ & 3 & \begin{tabular}{|l|}
283.0738 \\
\end{tabular} & 2.7E-04 & 3 & \begin{tabular}{|l|}
283.0695 \\
\end{tabular} & unknown & unknown \\
\hline $2.9 \mathrm{E}-06$ & 3 & \begin{tabular}{|l|}
104.1081 \\
\end{tabular} & $1.2 \mathrm{E}-04$ & 4 & \begin{tabular}{|l|}
104.1073 \\
\end{tabular} & $\mathrm{C} 5 \mathrm{H} 14 \mathrm{NO}+\mathrm{H}$ & Choline \\
\hline $2.4 \mathrm{E}-06$ & 3 & \begin{tabular}{|l|}
299.0473 \\
\end{tabular} & $1.2 \mathrm{E}-04$ & 5 & \begin{tabular}{|l|}
299.0437 \\
\end{tabular} & unknown & unknown \\
\hline $2.0 \mathrm{E}-07$ & 3 & \begin{tabular}{|l|}
261.0911 \\
\end{tabular} & $4.0 \mathrm{E}-04$ & 3 & \begin{tabular}{|l|}
261.0877 \\
\end{tabular} & unknown & unknown \\
\hline $1.3 \mathrm{E}-05$ & 3 & \begin{tabular}{|l|}
678.5107 \\
\end{tabular} & $8.4 \mathrm{E}-05$ & 4 & \begin{tabular}{|l|}
678.5065 \\
\end{tabular} & $\mathrm{C} 36 \mathrm{H} 72 \mathrm{NO} 8 \mathrm{P}+\mathrm{H}$ & $P C(28: 0)$ \\
\hline $3.0 \mathrm{E}-05$ & 3 & \begin{tabular}{|l|}
351.2481 \\
\end{tabular} & $9.4 \mathrm{E}-05$ & 5 & \begin{tabular}{|l|}
351.2502 \\
\end{tabular} & $\mathrm{C} 19 \mathrm{H} 3604+\mathrm{Na}$ & MG (16:1) \\
\hline $2.8 \mathrm{E}-05$ & 3 & \begin{tabular}{|l|}
123.0564 \\
\end{tabular} & $9.8 \mathrm{E}-05$ & 2 & \begin{tabular}{|l|}
123.0554 \\
\end{tabular} & $\mathrm{C} 6 \mathrm{H} 6 \mathrm{~N} 2 \mathrm{O}+\mathrm{H}$ & Niacinamide \\
\hline $1.6 \mathrm{E}-07$ & 3 & \begin{tabular}{|l|}
203.1517 \\
\end{tabular} & $3.8 \mathrm{E}-04$ & 6 & \begin{tabular}{|l|}
203.1501 \\
\end{tabular} & $\mathrm{C} 8 \mathrm{H} 18 \mathrm{~N} 4 \mathrm{O} 2+\mathrm{H}$ & Dimethylarginine \\
\hline $6.2 \mathrm{E}-06$ & 3 & \begin{tabular}{|l|}
261.0642 \\
\end{tabular} & $9.8 \mathrm{E}-05$ & 2 & \begin{tabular}{|l|}
261.0579 \\
\end{tabular} & unknown & unknown \\
\hline $6.7 \mathrm{E}-03$ & 2 & \begin{tabular}{|l|}
704.5222 \\
\end{tabular} & $3.6 \mathrm{E}-06$ & 2 & \begin{tabular}{|l|}
704.5226 \\
\end{tabular} & $\mathrm{C} 38 \mathrm{H} 74 \mathrm{NO} 8 \mathrm{P}+\mathrm{H}$ & $P C(30: 1)$ \\
\hline $8.2 \mathrm{E}-06$ & 2 & \begin{tabular}{|l|}
258.1129 \\
\end{tabular} & $7.0 \mathrm{E}-04$ & 7 & \begin{tabular}{|l|}
258.1099 \\
\end{tabular} & $\mathrm{C} 8 \mathrm{H} 2 \mathrm{ONO} 6 \mathrm{P}+\mathrm{H}$ & Glycerophos phocholine \\
\hline $1.7 \mathrm{E}-04$ & 2 & \begin{tabular}{|l|}
518.3296 \\
\end{tabular} & $5.7 \mathrm{E}-04$ & 3 & 518.3218 & $\mathrm{C} 24 \mathrm{H} 50 \mathrm{NO} 07 \mathrm{P}+\mathrm{Na}, \mathrm{C} 26 \mathrm{H} 48 \mathrm{NO} \mathrm{PP}+\mathrm{H}$ & LysoPC(16:0) Na, LysoPC(18:3) \\
\hline $7.1 \mathrm{E}-06$ & 2 & \begin{tabular}{|l|}
247.0451 \\
\end{tabular} & $3.1 \mathrm{E}-05$ & 2 & \begin{tabular}{|l|}
247.0422 \\
\end{tabular} & unknown & unknown \\
\hline $1.2 \mathrm{E}-04$ & 2 & \begin{tabular}{|l|}
522.3579 \\
\end{tabular} & $6.5 \mathrm{E}-04$ & 1 & 522.3554 & $\mathrm{C} 26 \mathrm{H} 52 \mathrm{NO} 7 \mathrm{P}+\mathrm{H}$ & LysoPC(18:1) \\
\hline $1.2 \mathrm{E}-04$ & 2 & \begin{tabular}{|l|}
159.0295 \\
\end{tabular} & $8.7 \mathrm{E}-05$ & 2 & \begin{tabular}{|l|}
159.0276 \\
\end{tabular} & $\mathrm{C} 5 \mathrm{H} 4 \mathrm{~N} 4 \mathrm{O}+\mathrm{Na}$ & Hypoxanthine \\
\hline $3.7 \mathrm{E}-06$ & 2 & \begin{tabular}{|l|}
137.0478 \\
\end{tabular} & $5.6 \mathrm{E}-05$ & 1 & \begin{tabular}{|l|}
137.0458 \\
\end{tabular} & $\mathrm{C} 5 \mathrm{H} 4 \mathrm{~N} 4 \mathrm{O}+\mathrm{H}$ & Hypoxanthine \\
\hline $3.5 \mathrm{E}-04$ & 2 & \begin{tabular}{|l|}
544.3386 \\
\end{tabular} & $2.4 \mathrm{E}-03$ & 2 & 544.3374 & $\mathrm{C} 26 \mathrm{H} 52 \mathrm{NO} \mathrm{PP}+\mathrm{Na}, \mathrm{C} 28 \mathrm{H} 50 \mathrm{NO} \mathrm{P}+\mathrm{H}$ & LysoPC(18:1) Na + LysoPC(20:4) \\
\hline $8.9 \mathrm{E}-07$ & 1 & \begin{tabular}{|l|}
206.0575 \\
\end{tabular} & $1.7 \mathrm{E}-04$ & 3 & \begin{tabular}{|l|}
206.0552 \\
\end{tabular} & $\mathrm{C} 5 \mathrm{H} 15 \mathrm{NO} 4 \mathrm{P}+\mathrm{Na}$ & Phosphorylcholine \\
\hline $3.6 \mathrm{E}-06$ & 1 & \begin{tabular}{|l|}
147.1143 \\
\end{tabular} & $1.1 \mathrm{E}-04$ & 1 & 147.1128 & $\mathrm{C} 6 \mathrm{H} 14 \mathrm{~N} 2 \mathrm{O} 2+\mathrm{H}$ & L-Lysine \\
\hline $1.4 \mathrm{E}-04$ & 1 & \begin{tabular}{|l|}
169.0605 \\
\end{tabular} & $1.6 \mathrm{E}-05$ & 1 & 169.0583 & $\mathrm{C} 5 \mathrm{H} 10 \mathrm{~N} 2 \mathrm{O} 3+\mathrm{Na}$ & L-Glutamine \\
\hline $6.7 E-05$ & 1 & \begin{tabular}{|l|}
175.0024 \\
\end{tabular} & $3.5 E-04$ & 1 & 175.0016 & $\mathrm{C} 5 \mathrm{H} 4 \mathrm{~N} 4 \mathrm{O}+\mathrm{K}$ & Hypoxanthine \\
\hline $7.9 \mathrm{E}-07$ & 1 & \begin{tabular}{|l|}
347.0317 \\
\end{tabular} & $1.1 \mathrm{E}-03$ & 2 & \begin{tabular}{|l|}
347.0249 \\
\end{tabular} & $\mathrm{C} 9 \mathrm{H} 13 \mathrm{~N} 2 \mathrm{O} \mathrm{PP}+\mathrm{Na}$ & UMP \\
\hline $1.6 \mathrm{E}-06$ & 1 & \begin{tabular}{|l|}
118.0873 \\
\end{tabular} & $5.6 \mathrm{E}-05$ & 1 & 118.0865 & $\mathrm{C} 5 \mathrm{H} 11 \mathrm{NO} 2+\mathrm{H}$ & Betaine \\
\hline $6.3 \mathrm{E}-07$ & 1 & \begin{tabular}{|l|l|}
780.5554 \\
\end{tabular} & $6.9 \mathrm{E}-03$ & 2 & 780.5511 & $\mathrm{C} 42 \mathrm{H} 80 \mathrm{NO} O 8 \mathrm{P}+\mathrm{Na}, \mathrm{C} 44 \mathrm{H} 78 \mathrm{NO} \mathrm{PP}+\mathrm{H}$ & $P C(32: 2) \mathrm{Na}, \mathrm{PC}(36: 5)$ \\
\hline $2.3 \mathrm{E}-06$ & 1 & \begin{tabular}{|l|}
147.0787 \\
\end{tabular} & $1.0 \mathrm{E}-04$ & 1 & \begin{tabular}{|l|}
147.0764 \\
\end{tabular} & $\mathrm{C} 5 \mathrm{H} 10 \mathrm{~N} 2 \mathrm{O} 3+\mathrm{H}$ & L-Glutamine \\
\hline 6.6E-05 & 1 & \begin{tabular}{|l|}
184.0754 \\
\end{tabular} & $1.2 \mathrm{E}-04$ & 2 & 184.0733 & $\mathrm{C} 5 \mathrm{H} 15 \mathrm{NO} 4 \mathrm{P}+\mathrm{H}$ & Phosphorylcholine \\
\hline 7.1E-05 & 1 & \begin{tabular}{|l|}
305.0047 \\
\end{tabular} & $4.2 \mathrm{E}-04$ & 2 & 305.0006 & unknown & unknown \\
\hline 1.6E-04 & -1 & \begin{tabular}{|l|}
748.5258 \\
\end{tabular} & $1.2 \mathrm{E}-03$ & -5 & 748.5269 & $\mathrm{C} 43 \mathrm{H} 74 \mathrm{NO} 07 \mathrm{P}+\mathrm{H}, \mathrm{C} 41 \mathrm{H} 76 \mathrm{NO} \mathrm{P}+\mathrm{Na}$ & $\operatorname{PE}(38: 7 e), \operatorname{PE}(36: 4 \mathrm{e}) \mathrm{Na}$ \\
\hline 2.2E- 04 & -1 & \begin{tabular}{|l|}
718.5392 \\
\end{tabular} & $7.8 \mathrm{E}-04$ & -3 & 718.5381 & $\mathrm{C} 39 \mathrm{H} 76 \mathrm{NO} 08 \mathrm{P}+\mathrm{H}$ & PE(34:1) \\
\hline 4.2E-05 & -2 & 754.5318 & $5.6 \mathrm{E}-03$ & 2 & 754.5355 & $\mathrm{C} 40 \mathrm{H} 78 \mathrm{NO} 8 \mathrm{P}+\mathrm{Na}, \mathrm{C} 42 \mathrm{H} 76 \mathrm{NO} \mathrm{PP}+\mathrm{H}$ & $\mathrm{PC}(32: 1) \mathrm{Na}, \mathrm{PC}(34: 4)$ \\
\hline 4.5E-05 & -2 & 834.5949 & $8.4 \mathrm{E}-03$ & 1 & 834.5986 & $\mathrm{C} 46 \mathrm{H} 86 \mathrm{NO} O \mathrm{P}+\mathrm{Na}, \mathrm{C} 48 \mathrm{H} 84 \mathrm{NO} \mathrm{OP}+\mathrm{H}$ & $P C(38: 3) \mathrm{Na}, \mathrm{PC}(40: 6)$ \\
\hline $8.8 \mathrm{E}-06$ & -3 & 132.0785 & $1.6 \mathrm{E}-04$ & -3 & 132.0768 & $\mathrm{C} 4 \mathrm{H} 9 \mathrm{~N} 3 \mathrm{O} 2+\mathrm{H}$ & Creatine \\
\hline 1.2E-04 & -4 & 746.5673 & $1.2 \mathrm{E}-03$ & -3 & 746.569 & $\mathrm{C} 41 \mathrm{H} 80 \mathrm{NO} \mathrm{PP}+\mathrm{H}$ & $\operatorname{PE}(36: 1), P C(33: 1)$ \\
\hline $3.0 \mathrm{E}-08$ & -4 & 830.5710 & $6.8 \mathrm{E}-06$ & -2 & 830.5663 & $\mathrm{C} 46 \mathrm{H} 82 \mathrm{NO} 8 \mathrm{P}+\mathrm{Na}, \mathrm{C} 48 \mathrm{H} 80 \mathrm{NO} O \mathrm{P}+\mathrm{H}$ & $P C(38: 5) \mathrm{Na}, \mathrm{PC}(40: 8)$ \\
\hline $1.4 \mathrm{E}-06$ & -4 & 772.5765 & $9.7 E-04$ & -3 & 772.5839 & $\mathrm{C} 43 \mathrm{H} 82 \mathrm{NO} 08 \mathrm{P}+\mathrm{H}$ & $\mathrm{PE}(38: 2)$ \\
\hline $3.9 \mathrm{E}-09$ & -5 & 856.5875 & $1.1 \mathrm{E}-06$ & -3 & 856.5813 & $\mathrm{C} 48 \mathrm{H} 84 \mathrm{NO} 8 \mathrm{P}+\mathrm{Na}, \mathrm{C} 50 \mathrm{H} 82 \mathrm{NO} \mathrm{PP}+\mathrm{H}$ & $P C(40: 6) \mathrm{Na}, \mathrm{PC}(42: 9)$ \\
\hline $1.7 \mathrm{E}-05$ & -5 & 162.1139 & $1.7 \mathrm{E}-04$ & -4 & 162.1124 & $\mathrm{C} 7 \mathrm{H} 15 \mathrm{NO} 3+\mathrm{H}$ & L-carnitine \\
\hline $5.9 \mathrm{E}-04$ & -6 & 818.5975 & $2.7 \mathrm{E}-03$ & 3 & 818.6029 & $\mathrm{C} 46 \mathrm{H} 86 \mathrm{NO} 07 \mathrm{P}+\mathrm{Na}, \mathrm{C} 48 \mathrm{H} 84 \mathrm{NO} \mathrm{P}+\mathrm{H}$ & $P C(38: 4 e) \mathrm{Na}, \mathrm{PC}(40: 7 \mathrm{e})$ \\
\hline $9.0 \mathrm{E}-07$ & -8 & \begin{tabular}{|l|}
137.0702 \\
\end{tabular} & $1.2 \mathrm{E}-04$ & -4 & 137.071 & $\mathrm{C} 7 \mathrm{H} 8 \mathrm{~N} 2 \mathrm{O}+\mathrm{H}$ & N-Methylnicotinamide \\
\hline $1.9 \mathrm{E}-04$ & -8 & \begin{tabular}{|l|}
828.5468 \\
\end{tabular} & $3.6 \mathrm{E}-06$ & -5 & 828.5505 & $\mathrm{C} 46 \mathrm{H} 80 \mathrm{NO} O 8 \mathrm{P}+\mathrm{Na}, \mathrm{C} 48 \mathrm{H} 78 \mathrm{NO} \mathrm{OP}+\mathrm{H}$ & $\mathrm{PC}(38: 6) \mathrm{Na}, \mathrm{PC}(40: 9)$ \\
\hline
\end{tabular}

